\title{
Games of Threats
}

\section{Citation}

Kohlberg, Elon, and Abraham Neyman. "Games of Threats." Games and Economic Behavior 108 (March 2018): 139-145.

\section{Permanent link}

http://nrs.harvard.edu/urn-3:HUL.InstRepos:39148383

\section{Terms of Use}

This article was downloaded from Harvard University's DASH repository, and is made available under the terms and conditions applicable to Open Access Policy Articles, as set forth at http:// nrs.harvard.edu/urn-3:HUL.InstRepos:dash.current.terms-of-use\#OAP

\section{Share Your Story}

The Harvard community has made this article openly available.

Please share how this access benefits you. Submit a story.

Accessibility 


\title{
GAMES OF THREATS
}

\author{
ELON KOHLBERG* AND ABRAHAM NEYMAN ${ }^{+}$
}

\begin{abstract}
A game of threats on a finite set of players, $N$, is a function $d$ that assigns a real number to any coalition, $S \subseteq N$, such that $d(S)=-d(N \backslash S)$. A game of threats is not necessarily a coalitional game as it may fail to satisfy the condition $d(\emptyset)=0$. We show that analogs of the classic Shapley axioms for coalitional games determine a unique value for games of threats. This value assigns to each player an average of $d(S)$ across all the coalitions that include the player. Games of threats arise naturally in value theory for strategic games, and may have applications in other branches of game theory.
\end{abstract}

\section{INTRODUCTION}

The Shapley value is the most widely studied solution concept of cooperative game theory. It is defined on coalitional games, which are the standard objects of the theory.

A coalitional game on a finite set of players, $N$, is a function $v$ that assigns a real number to any subset ("coalition"), $S \subseteq N$, such that $v(\emptyset)=0$. The amount $v(S)$ may be interpreted as the worth of $S$, i.e., what the players belonging to $S$ can jointly get by coordinating their efforts.

A value is a function that assigns to each coalitional game a vector of payoffs, one for each player, that reflects the a priori evaluation of each player's position in the game. In his celebrated paper [4] Shapley proposed four desirable properties ("axioms") and proved the remarkable result that there exists a unique function satisfying these properties. This function, the Shapley value, can be described as follows. The value of player $i$ is an average of the marginal contributions, $v(S \cup i)-v(S)$, of player $i$, where the average is taken over all the orderings of $N$, with $S$ denoting the subset of players that precede $i$ in the ordering.

A game of threats is a function $d$ that assigns a real number to any coalition, $S \subseteq N$, such that $d(S)=-d(N \backslash S)$. The amount $d(S)$ may be interpreted as the threat power of the coalition $S$, i.e., the maximal difference between the total amounts that the players belonging to $S$ and the players belonging to $N \backslash S$ receive, when the players in $S$ coordinate their efforts to maximize this difference and the players in $N \backslash S$ coordinate their efforts to minimize it. Games of threats arise naturally in value theory for strategic games [2].

Date: October 23, 2017.

* Harvard Business School, Harvard University; ekohlberg@hbs.edu.

+ Institute of Mathematics, and the Federmann Center for the Study of Rationality, The Hebrew University of Jerusalem, Givat Ram, Jerusalem 91904, Israel; aneyman@math.huji.ac.il. 
There, the condition $d(S)=-d(N \backslash S)$ is a consequence of the minmax theorem; see the Appendix.

Now, games of threats need not be coalitional games, as they may fail to satisfy the condition $d(\emptyset)=0$. Thus, if we wish to obtain an a priori evaluation for games of threats then we must develop a value theory for such games. This paper does that. We show that there is a unique function, from games of threats to $n$-dimensional payoff vectors, that satisfies the analogs of Shapley's four axioms; and that this function can be described as follows. The value of a player is the average of the threat powers, $d(S)$, of the coalitions that include the player. Specifically, if $d_{i, k}$ denotes the average of $d(S)$ over all $k$-player coalitions that include $i$, then the value of player $i$ is the average of $d_{i, k}$ over $k=1,2, \ldots, n$.

We take three approaches: a derivation of the results from classic Shapley value theory for coalitional games, a direct derivation based on the formula, and a direct derivation based on the random-order approach. This last derivation establishes a formula for the Shapley value of games of threats that is analogous to the formula for coalitional games: the value of player $i$ is the average of $d(S \cup i)$, where the average is taken over all the orderings of $N$, with $S$ denoting the subset of players that precede $i$ in the ordering.

We end this introduction by noting that the existence and uniqueness of the Shapley value for games of threats is an essential component of the proof of the existence and uniqueness of a value for strategic games [2].

\section{Games of Threats - Definition}

A coalitional game of threats is a pair $(N, d)$, where

- $N=\{1, \ldots, n\}$ is a finite set of players.

- $d: 2^{N} \rightarrow \mathbb{R}$ is a function such that $d(S)=-d(N \backslash S)$ for all $S \subseteq N$.

\section{Example:}

$N=\{1,2,3\}$.

$d(\emptyset)=-1, d(1)=d(2)=-1, d(3)=0, d(1,2)=0, d(1,3)=d(2,3)=1, d(1,2,3)=1$.

Denote by $\mathbb{D}(N)$ the set of all coalitional games of threats. By choosing, for every $S \subseteq N$, either $S$ or $N \backslash S$, we can describe any $d \in \mathbb{D}(N)$ by means of $2^{n-1}$ numbers, thereby identifying $\mathbb{D}(N)$ with $\mathbb{R}^{2^{n-1}}$. One convenient choice is $(d(S))_{S \ni 1}$.

With this choice, the above example is described as follows.

$$
d(1)=-1, d(1,2)=0, d(1,3)=1, d(1,2,3)=1 .
$$

\section{The Shapley Value}

Let $\psi: \mathbb{D}(N) \rightarrow \mathbb{R}^{n}$ be a map that associates with each game of threats an allocation of payoffs to the players. Following Shapley [4], we consider the following axioms.

For all games of threats $(N, d),(N, e)$, for all players $i, j$, and for all real numbers $\alpha, \beta$ (and using the notation $\psi_{i} d$ for $\psi(d)(i)$ ), the following properties hold: 
- Efficiency $\sum_{i \in N} \psi_{i} d=d(N)$.

- Linearity $\psi(\alpha d+\beta e)=\alpha \psi d+\beta \psi e$.

- Symmetry $\psi_{i}(d)=\psi_{j}(d)$ if $i$ and $j$ are interchangeable ${ }^{1}$ in $d$ (i.e., if $d(S \cup i)=$ $d(S \cup j) \forall S \subseteq N \backslash\{i, j\})$.

- Null player $\psi_{i} d=0$ if $i$ is a null player in $d$ (i.e., if $d(S \cup i)=d(S) \forall S \subseteq N$ ).

Definition 1. A map $\psi: \mathbb{D}(N) \rightarrow \mathbb{R}^{n}$ satisfying the above axioms is called a value.

Theorem 1. There exists a unique value for games of threats. It may be described as follows:

$$
\psi_{i} d=\frac{1}{n} \sum_{k=1}^{n} d_{i, k}
$$

where $d_{i, k}$ denotes the average of $d(S)$ over all $k$-player coalitions that include $i$.

In the example, $\psi_{1}=\psi_{2}=\frac{1}{3} \times(-1)+\frac{1}{3} \times \frac{1+0}{2}+\frac{1}{3} \times 1=\frac{1}{6}, \quad \psi_{3}=\frac{1}{3} \times 0+\frac{1}{3} \times 1+\frac{1}{3} \times 1=\frac{2}{3}$.

Note: Formula (1) allocates to each player a weighted average of the $d(S)$ over the coalitions $S$ that include that player. The weight is the same for all coalitions of the same size but different for coalitions of a different size. Specifically, for each $k=1, \ldots, n$, the total weight of $\frac{1}{n}$ is divided among the $\left(\begin{array}{l}n-1 \\ k-1\end{array}\right)$ coalitions of size $k$ that include $i$ :

$$
\psi_{i} d=\frac{1}{n} \sum_{k=1}^{n} \frac{1}{\left(\begin{array}{c}
n-1 \\
k-1
\end{array}\right)} \sum_{\substack{S: i \in S \\
|S|=k}} d(S)=\frac{1}{n} \sum_{S: i \in S} \frac{1}{\left(\begin{array}{c}
n-1 \\
|S|-1
\end{array}\right)} d(S) .
$$

Note: Henceforth we shall refer to the map of equation 1 as the Shapley value for games of threats.

\section{Derivation from Classic Shapley value theory}

Let $\mathbb{V}(N):=\left\{v: 2^{N} \rightarrow R, v(\emptyset)=0\right\}$ be the set of standard coalitional games on $N$. It can be identified with a subspace of $\mathbb{R}^{2^{n}}$ of dimension $2^{n}-1$.

Let $K: \mathbb{V}(N) \rightarrow \mathbb{D}(N)$ and $L: \mathbb{D}(N) \rightarrow \mathbb{V}(N)$ be defined by

and

$$
(K v)(S)=v(S)-v(N \backslash S)
$$

$$
(L d)(S)=\frac{1}{2} d(S)+\frac{1}{2} d(N)
$$

\footnotetext{
${ }^{1}$ In the Shapley-value literature, such players are called substitutes rather than interchangeable. We adopt the latter term in order to avoid potential confusions that may arise from the standard meaning of the term "substitutes" in economics.
} 
Note that the map $L$ is linear, efficient (i.e., $L(d)(N)=d(N)$ ), symmetric (i.e., if $i$ and $j$ are interchangeable in $d \in \mathbb{D}(N)$ then $i$ and $j$ are interchangeable in $L d \in \mathbb{V}(N)$ ), and preserves null players (i.e., if $i$ is a null player in $d \in \mathbb{D}(N)$ then $i$ is a null player in $L d \in \mathbb{V}(N))$.

Thus, if $\varphi$ is a value on $\mathbb{V}(N)$, then $\varphi \circ L$ is a value on $\mathbb{D}(N)$.

Note that $K \circ L$ is the identity on $\mathbb{D}(N)$; therefore - in particular - $K$ is surjective. It follows that, if $\psi_{1}$ and $\psi_{2}$ are two different values on $\mathbb{D}(N)$, then $\psi_{1} \circ K$ and $\psi_{2} \circ K$ are two different values on $\mathbb{V}(N)$.

Thus if the value on $\mathbb{V}(N)$ is unique then the value on $\mathbb{D}(N)$ is unique. As the Shapley value $\varphi$ is the unique value on $\mathbb{V}(N), \varphi \circ L$ is the unique value on $\mathbb{D}(N)$.

This completes the proof of the existence and uniqueness of a value on $\mathbb{D}(N)$.

In order to show that the map $\psi$ of Theorem 1 is, in fact, the Shapley value on $\mathbb{D}(N)$, it suffices to show that $\psi=\varphi \circ L$.

Recall that the Shapley value $\varphi$ on $\mathbb{V}(N)$ is defined as follows. If $v \in \mathbb{V}(N)$ then

$$
\varphi_{i} v:=\frac{1}{n !} \sum_{\mathcal{R}}\left(v\left(P_{i}^{\mathcal{R}} \cup i\right)-v\left(P_{i}^{\mathcal{R}}\right)\right),
$$

where the summation is over the $n$ ! orderings of the set $N$ and where $P_{i}^{\mathcal{R}}$ denotes the subset of those $j \in N$ that precede $i$ in the ordering $\mathcal{R}$. Let $d$ be a game of threats. Then, $(L d)\left(P_{i}^{\mathcal{R}} \cup i\right)-(L d)\left(P_{i}^{\mathcal{R}}\right)=\frac{1}{2} d\left(P_{i}^{\mathcal{R}} \cup i\right)-\frac{1}{2} d\left(P_{i}^{\mathcal{R}}\right)$. The complement of $P_{i}^{\mathcal{R}}$ is $P_{i}^{\mathcal{R}^{*}} \cup i$, where $\mathcal{R}^{*}$ denotes the reverse ordering of $\mathcal{R}$. As $d$ is a game of threats, we have $-\frac{1}{2} d\left(P_{i}^{\mathcal{R}}\right)=\frac{1}{2} d\left(P_{i}^{\mathcal{R}^{*}} \cup i\right)$. Therefore,

$$
\varphi_{i}(L d)=\frac{1}{n !} \sum_{\mathcal{R}}\left(\frac{1}{2} d\left(P_{i}^{\mathcal{R}} \cup i\right)+\frac{1}{2} d\left(P_{i}^{\mathcal{R}^{*}} \cup i\right)\right) .
$$

Since the set of reverse orderings is the same as the set of orderings,

$$
\varphi_{i}(L d)=\frac{1}{n !} \sum_{\mathcal{R}} d\left(P_{i}^{\mathcal{R}} \cup i\right)
$$

But

$$
\frac{1}{n !} \sum_{\mathcal{R}} d\left(P_{i}^{\mathcal{R}} \cup i\right)=\frac{1}{n} \sum_{k=1}^{n} \frac{1}{(n-1) !} \sum_{\mathcal{R}:\left|P_{i}^{\mathcal{R}}\right|=k-1} d\left(P_{i}^{\mathcal{R}} \cup i\right)=\frac{1}{n} \sum_{k=1}^{n} d_{i, k}
$$

Thus

$$
\varphi_{i}(L d)=\frac{1}{n} \sum_{k=1}^{n} d_{i, k}
$$

which completes the proof that $\psi=\varphi \circ L$. 
Remark: The map $K$ is linear, efficient, symmetric, and preserves null players. Thus, if $\psi$ is a value on $\mathbb{D}(N)$ then $\psi \circ K$ is a value on $\mathbb{V}(N)$. In particular, the existence of a value on $\mathbb{D}(N)$ implies the existence of a value on $\mathbb{V}(N))$.

Young [6] showed that the existence and uniqueness theorem for the Shapley value in $\mathbb{V}(N)$ remains valid when the axioms of linearity and null player are replaced by an axiom of marginality, which requires that the value of a player $i$ in a game $v$ depend only on the player's marginal contributions, $v(S \cup i)-v(S)$. Now, the map $L$ preserves marginality (i.e., if the marginal contributions of player $i$ are the same in two games $d_{1}, d_{2} \in \mathbb{D}(N)$ then the marginal contributions are the same in $\left.L d_{1}, L d_{2} \in \mathbb{V}(N)\right)$; therefore the same argument as above implies the following.

Corollary 1. The Shapley value is the unique mapping $\psi: \mathbb{D}(N) \rightarrow \mathbb{R}^{n}$ satisfying the axioms of efficiency, symmetry, and marginality.

Remark: The map $K$ also satisfies marginality. Thus the existence of a value satisfying Young's axioms on $\mathbb{D}(N)$ implies the existence of such a value on $\mathbb{V}(N))$.

We end this section by noting that the results for games of threats are also valid for constant-sum games.

Let $\mathbb{C}(N):=\left\{v: 2^{N} \rightarrow R, \quad v(S)+v(N \backslash S)=v(N)\right.$ for all $\left.S \subseteq N\right\}$ be the set of constant-sum coalitional games on $N$. It can be viewed as a subspace of $\mathbb{R}^{2^{n}}$ of dimension $2^{n-1}$.

Let $K^{\prime}: \mathbb{V}(N) \rightarrow \mathbb{C}(N)$ and $L^{\prime}: \mathbb{C}(N) \rightarrow \mathbb{V}(N)$ be defined by

$$
\left(K^{\prime} v\right)(S)=\frac{1}{2}(v(S)-v(N \backslash S)+v(N))
$$

and

$$
\left(L^{\prime} c\right)(S)=c(S)
$$

Then both $L^{\prime}$ and $K^{\prime}$ are linear, efficient, symmetric, preserve null players, and preserve marginality, and $K^{\prime} \circ L^{\prime}$ is the identity on $\mathbb{C}(N)$. This implies the existence and uniqueness of a value on $\mathbb{C}(N)$ in exactly the same way that the parallel statement for $\mathbb{D}(N)$ implied the existence and uniqueness of a value on $\mathbb{D}(N)$.

Corollary 2. The Shapley value is the unique mapping $\psi: \mathbb{C}(N) \rightarrow \mathbb{R}^{n}$ satisfying the axioms of linearity, efficiency, symmetry, and null player, as well as the unique such mapping satisfying the axioms of efficiency, symmetry, and marginality.

\section{Direct DeRivation from the Formula of Theorem 1}

Definition 2. Let $T \subseteq N, T \neq \emptyset$. The unanimity game, $u_{T} \in \mathbb{D}(N)$, is defined by 


$$
u_{T}(S)=\left\{\begin{aligned}
|T| & \text { if } S \supseteq T, \\
-|T| & \text { if } S \subseteq N \backslash T \\
0 & \text { otherwise. }
\end{aligned}\right.
$$

Proposition 2. The unanimity games span $\mathbb{D}(N)$.

Proof. It is sufficient to show that the $2^{n-1}$ games $\left(u_{T}\right)_{T \ni 1}$ are linearly independent. Suppose, then, that $\sum a_{j} u_{T_{j}}=0$, where $T_{i} \neq T_{j}$ for $i \neq j$ and not all the $a_{j}$ are zero.

Since, for $i \neq j, T_{i} \neq T_{j}$ and $T_{i} \cap T_{j} \supseteq\{1\} \neq \emptyset$, neither set is contained in the other's complement and therefore

$$
u_{T_{j}}\left(T_{i}\right)= \begin{cases}\left|T_{j}\right| & \text { if } T_{i} \supseteq T_{j} \\ 0 & \text { otherwise }\end{cases}
$$

Among the $T_{j}$ for which the coefficient $a_{j}$ is non-zero choose one, say $T_{1}$, with a minimum number of players. Then for any $j>1, T_{1} \nsupseteq T_{j}$ and therefore, by $(5), u_{T_{j}}\left(T_{1}\right)=0$. Thus $0=\sum a_{j} u_{T_{j}}\left(T_{1}\right)=a_{1} u_{T_{1}}\left(T_{1}\right)=a_{1}\left|T_{1}\right| \neq 0$, a contradiction.

Proof of Theorem 1. In the unanimity game $u_{T}$, all $i \notin T$ are null players and all $i \in T$ are interchangeable. It follows that any map that satisfies the efficiency, symmetry, and null player axioms, is uniquely determined on $u_{T}$ :

$$
\psi_{i} u_{T}= \begin{cases}1 & \text { for } i \in T \\ 0 & \text { for } i \notin T\end{cases}
$$

If the map also satisfies linearity then - by Proposition 2 - it is determined on all of $\mathbb{D}(N)$. This establishes uniqueness.

It is easy to verify that the map $\psi$ defined in (1) satisfies linearity and symmetry. To verify that it satisfies the null player axiom, proceed as follows. Consider the basic condition $d(S)=-d(N \backslash S)$. As $S$ ranges over all sets of size $k$ that include $i, N \backslash S$ ranges over all sets of size $n-k$ that do not include $i$. Averaging over all these sets, we have

$$
d_{i, k}=-\underline{d}_{i, n-k},
$$

where $\underline{d}_{i, k}$ denotes the average of $d(S)$ over all $k$-player sets that do not include $i$.

Now, if $i$ is a null player then $d(S)=d(S \cup i)$ for any set $S$ that does not include $i$. Taking the average over all such sets $S$ of size $n-k$, we have $\underline{d}_{i, n-k}=d_{i, n-k+1}$, which combined with (7) - yields

$$
d_{i, k}=-d_{i, n-k+1}
$$

Summing over $k=1, \ldots, n$ and dividing by $n$ yields $\psi_{i} d=-\psi_{i} d$. Thus $\psi_{i} d=0$.

It remains to prove efficiency, namely, that

$$
\sum_{i=1}^{n} \psi_{i} d=d(N)
$$


Let $D_{k}$ denote the average of the $d(S)$ over all $k$-player coalitions. Since $d_{i, k}$ is the average of all the $d(S)$ with $i \in S$ and $|S|=k$, it follows by symmetry that the average of $d_{i, k}$ over $i=1, \ldots, n$ is $D_{k}$. Thus

$$
\sum_{i=1}^{n} \psi_{i} d=\sum_{i=1}^{n} \frac{1}{n} \sum_{k=1}^{n} d_{i, k}=\sum_{k=1}^{n} \frac{1}{n} \sum_{i=1}^{n} d_{i, k}=\sum_{k=1}^{n} D_{k} .
$$

Note that there is just one $n$-player coalition, namely, $N$, so $d_{N}=d(N)$. Thus, to prove (8), we must show that

$$
\sum_{k=1}^{n-1} D_{k}=0
$$

But this follows from the fact that

$$
D_{k}=-D_{n-k} \text { for } k=1, \ldots, n-1
$$

which in turn follows from the basic condition $d(S)=-d(N \backslash S)$, as seen by noting that as we take an average of the left side over all the sets of size $k$, the right side is averaged over all the sets of size $n-k$.

Note: The theorem remains valid when the axiom of linearity is replaced by the weaker axiom of additivity. The proof is the same, with the additional observation that efficiency, symmetry, and the null player axioms determine the value not only on the unanimity games but also on any multiple, $c u_{T}$, of such games.

\section{Direct derivation by the Random Order Approach}

In this section we provide an alternative formulation of Theorem 1 that is analogous to Shapley's classic formulation.

Proposition 3. There is a unique value for games of threats. It may be described as follows:

$$
\psi_{i} d=\frac{1}{n !} \sum_{\mathcal{R}} d\left(P_{i}^{\mathcal{R}} \cup i\right),
$$

where the summation is over the $n$ ! possible orderings of the set $N$ and where $P_{i}^{\mathcal{R}}$ denotes the subset of those $j \in N$ that precede $i$ in the ordering $\mathcal{R}$.

Proof. Equation (4) establishes that $\frac{1}{n !} \sum_{\mathcal{R}} d\left(P_{i}^{\mathcal{R}} \cup i\right)=\frac{1}{n} \sum_{k=1}^{n} d_{i, k}$. Therefore, by Theorem 1, the map $\psi$ defined in (9) is the unique Shapley value for games of threats.

In the rest of this section we present a direct proof of the proposition, without relying on Theorem 1.

The proof of uniqueness is the same as before. First, observe that the unanimity games span all games of threats and that the axioms uniquely determine the value for the unanimity games; then apply linearity. 
Next, we must prove that the map $\psi$ of equation (9) satisfies Shapley's four axioms. Linearity and symmetry are easy to verify. To prove efficiency, proceed as follows. Let $S_{j}^{\mathcal{R}}$ be the set of the first $j$ elements in the ordering $\mathcal{R}$. Then, for every $j, \frac{1}{n !} \sum_{\mathcal{R}} d\left(S_{j}^{\mathcal{R}}\right)=D_{j}$. As shown earlier, $D_{j}+D_{n-j}=0$ for $1 \leq j<n$. Therefore,

$$
\begin{aligned}
\sum_{i=1}^{n} \psi_{i} d & =\sum_{i=1}^{n} \frac{1}{n !} \sum_{\mathcal{R}} d\left(P_{i}^{\mathcal{R}} \cup i\right)=\frac{1}{n !} \sum_{\mathcal{R}} \sum_{i=1}^{n} d\left(P_{i}^{\mathcal{R}} \cup i\right) \\
& =\frac{1}{n !} \sum_{\mathcal{R}} \sum_{j=1}^{n} d\left(S_{j}^{\mathcal{R}}\right)=\sum_{j=1}^{n} D_{j}=D_{n}=d(N) .
\end{aligned}
$$

Next, we must prove that the map $\psi$ of equation (9) satisfies Shapley's four axioms. Linearity and symmetry are easy to verify. To prove efficiency, proceed as follows. Let $S_{j}^{\mathcal{R}}$ be the set of the first $j$ elements in the ordering $\mathcal{R}$. Then, for every $j, \frac{1}{n !} \sum_{\mathcal{R}} d\left(S_{j}^{\mathcal{R}}\right)=D_{j}$. As shown earlier, $D_{j}+D_{n-j}=0$ for $1 \leq j<n$. Therefore,

$$
\begin{aligned}
\sum_{i=1}^{n} \psi_{i} d & =\sum_{i=1}^{n} \frac{1}{n !} \sum_{\mathcal{R}} d\left(P_{i}^{\mathcal{R}} \cup i\right)=\frac{1}{n !} \sum_{\mathcal{R}} \sum_{i=1}^{n} d\left(P_{i}^{\mathcal{R}} \cup i\right) \\
& =\frac{1}{n !} \sum_{\mathcal{R}} \sum_{j=1}^{n} d\left(S_{j}^{\mathcal{R}}\right)=\sum_{j=1}^{n} D_{j}=D_{n}=d(N) .
\end{aligned}
$$

It remains to verify the null player axiom. If $i$ is a null player then $d\left(P_{i}^{\mathcal{R}} \cup i\right)=-d\left(P_{i}^{\mathcal{R}^{*}}\right)=$ $-d\left(P_{i}^{\mathcal{R}^{*}} \cup i\right)$. As $\mathcal{R}$ ranges over all the orderings, so does $\mathcal{R}^{*}$. Therefore,

$$
\psi_{i} d=\frac{1}{n !} \sum_{\mathcal{R}} d\left(P_{i}^{\mathcal{R}} \cup i\right)=-\frac{1}{n !} \sum_{\mathcal{R}^{*}} d\left(P_{i}^{\mathcal{R}^{*}} \cup i\right)=-\psi_{i} d,
$$

which implies that $\psi_{i} d=0$.

\section{Appendix A. The value of Strategic games}

A strategic game is a triple $G=(N, A, g)$, where

- $N=\{1, \ldots, n\}$ is a finite set of players,

- $A^{i}$ is the finite set of player $i$ 's pure strategies, and

- $g=\left(g^{i}\right)_{i \in N}$, where $g^{i}: \prod_{i \in S} A^{i} \rightarrow \mathbb{R}$ is player $i$ 's payoff function.

Denote by $X^{S}$ the probability distributions on $\prod_{i \in S} A^{i}$; these are the correlated strategies of the players in $S$.

Let $G \in \mathbb{G}(N)$. Define the threat power of coalition $S$ as follows: ${ }^{2}$

$$
(\delta G)(S):=\max _{x \in X^{S}} \min _{y \in X^{N \backslash S}}\left(\sum_{i \in S} g^{i}(x, y)-\sum_{i \notin S} g^{i}(x, y)\right) .
$$

\footnotetext{
${ }^{2}$ Expressions of the form max or min over the empty set are ignored.
} 
By the minmax theorem, $(\delta G)(S)=-(\delta G)(N \backslash S)$ for any $S \subseteq N$. Thus $\delta G$ is a game of threats.

We define the value, $\gamma G$, of the strategic game $G$ by taking the Shapley value of the game of threats $\delta G$.

In [2] it is proved that $\gamma$ is the unique function that satisfies five properties that are desirable in a map providing an a priori evaluation of the position of each player in a strategic game. Four of these properties are analogs of the Shapley axioms for the value of cooperative games.

Formula (1) then implies the following: the value of a player in an $n$-person strategic game $G$ is an average of the threat powers, $(\delta G)(S)$, of the subsets of which the player is a member. Specifically, if $\delta_{i, k}$ denotes the average of $(\delta G)(S)$ over all $k$-player coalitions that include $i$, then the value of player $i$ is the average of $\delta_{i, k}$ over $k=1,2, \ldots, n$.

Remark: The game of threats associated with a strategic game $G$, as defined in (12), is different from von Neumann and Morgenstern's original coalitional game, which is defined as follows:

$$
(v G)(S):=\max _{x \in X^{S}} \min _{y \in X^{N \backslash S}} \sum_{i \in S} g^{i}(x, y) .
$$

As was pointed out by von Neumann and Morgenstern [5], Shapley [3], Harsanyi [1], and others, this definition has some deficiencies. In the context of the Shapley value, the shift to definition (12), which was first proposed by Harsanyi [1], has proven successful in addressing these deficiencies. We wonder whether, in the context of other cooperative solution concepts, e.g., the stable set or the core, the deficiencies could similarly be addressed by a shift to games of threats or to other variants of the von Neumann-Morgenstern coalitional game.

\section{REFERENCES}

[1] Harsanyi, J. (1963), A Simplified Bargaining Model for the $n$-Person Cooperative Game, International Economic Review, 4, 194-220.

[2] Kohlberg, E. and A. Neyman (2017), Cooperative Strategic Games, Federmann Center for the Study of Rationality discussion paper 706 .

[3] Shapley, L. (1951), Notes on the $n$-Person Game - II: the Value of an $n$-Person Game, RAND research memorandum ATI 210720.

[4] Shapley, L. (1953), A Value for $n$-Person Games. In Kuhn, H.W. and Tucker, A.W. (eds.), Contributions to the Theory of Games, Annals of Mathematics Studies, 28, pp. 307-319.

[5] von Neumann, J. and O. Morgenstern (1953), Theory of Games and Economic Behavior, third edition, Princeton University Press.

[6] Young H.P. (1985), Monotonic Solutions of Cooperative Games, International Journal of Game Theory, 14, 65-72. 